\title{
Conocimientos, actitudes y barreras respecto a la gestión de aguas residuales en el sector comercial de la ciudad de La Libertad, El Salvador Knowledge, attitudes and barriers regarding wastewater management in the commercial sector of the city of La Libertad, El Salvador
}

Francisco Rosales-Ayala ${ }^{1}$, Rooel Campos-Rodríguez ${ }^{2}$, Cristian Moreira-Segura ${ }^{3}$

Rosales-Araya, F; Campos-Rodríguez, R; Moreira-Segura, C. Conocimientos, actitudes y barreras respecto a la gestión de aguas residuales en el sector comercial de la ciudad de La Libertad, El Salvador. Tecnología en Marcha. Vol. 33-1.

Enero-Marzo 2020. Pág 111-121.

doi) https://doi.org/10.18845/tm.v33i1.5026

1 Investigador. Universidad Estatal a Distancia. Costa Rica. Correo electrónico: francisco.rosales@attecs.com.

2 Doctor en Ciencias Naturales para el Desarrollo, profesor-investigador de la Escuela de Agronegocios. Tecnológico de Costa Rica. Costa Rica. Correo electrónico: rocampos@tec.ac.cr.

3 Doctor en Ciencias Naturales para el Desarrollo, profesor-investigador del Doctorado en Ciencias Naturales para el Desarrollo (DOCINADE). Instituto Tecnológico de Costa Rica. Costa Rica. Correo electrónico: cmoreira@tec.ac.cr. 


\title{
Palabras clave
}

Agua residual especial; actitudes; barreras; conocimientos; competencias.

\section{Resumen}

El objetivo de este artículo fue valorar los conocimientos, actitudes y barreras ambientales, que presentan las personas que se dedican a actividades comerciales de micro y pequeña empresa (MYPES) en la Ciudad portuaria La Libertad, en El Salvador, respecto a la Gestion Integral de las aguas residuales.

El ente encargado de la promoción y el desarrollo de programas de saneamiento ambiental, de acuerdo al código municipal es la administración municipal, quienes convencidos de que los procesos de educación ambiental son fundamentales para el desarrollo de su comunidad y en ausencia de un estudio de estas características, es que reconocen la importancia de esta investigación, para que sirva de punto de partida en procura de la gestión integral de las aguas residuales.

Se aplicaron encuestas a tres estratos fundamentales, Políticos, MYPES y Técnicos, como actores involucrados en el proceso. Las encuestas se validaron por criterio experto. La utilización de herramientas estadísticas para la selección de la muestra y comparación de los datos, estuvo presente. Una vez obtenida la información se sistematizó y organizo en conocimientos, actitudes, y barreras.

Los resultados muestran que los conocimientos son incipientes entre la muestra estudiada, por lo que se debe profundizar en estos, ya que, aunque existen actitudes pro ambientales, la existencia de barreras conceptuales, actitudinales y de control está repercutiendo en la sensibilización y el empoderamiento para llegar a realizar acciones ambientalmente positivas partiendo de las competencias de los estratos analizados.

\section{Keywords}

Special waste water; attitudes; barriers; knowledge; competencies.

\begin{abstract}
The aim of this article is to assess the knowledge, attitudes and environmental barriers, presented by people engaged in commercial activities of micro and small enterprises (MYPES) in the port city of La Libertad, El Salvador, regarding the Integral Management wastewater.

The entity responsible for the promotion and development of environmental sanitation programs, according to the municipal code, is the municipal administration, who are convinced that environmental education processes are fundamental for the development of their community and in the absence of a study of these characteristics, is that they recognize the importance of this research, so that it serves as a starting point for the integral management of wastewater

Surveys were applied to three fundamental strata, Politicians, MYPES and Technicians, as actors involved in the process. The surveys were validate by expert criteria. The use of statistical tools for the selection of the sample and comparison of the data was present. Once the information was obtain, it was systematize and organized into knowledge, attitudes, and barriers.

The results show that knowledge is incipient among the sample studied, so it should be deepened in these, since, although there are pro-environmental attitudes, the existence of conceptual, attitudinal and control barriers is having an impact on the awareness and empowerment for get to perform environmentally positive actions, starting from the competences of the strata analyzed.
\end{abstract}




\section{Situación de las Aguas Residuales}

Alrededor del mudo las aguas residuales se vierten al ambiente sin tratamiento alguno. Según [1], más del $80 \%$ de las aguas residuales (95\% en algunos países en desarrollo) se descargan directamente al cuerpo receptor, sin ningún proceso de depuración. Las consecuencias son diversas, por ejemplo, la mayoría de ríos de África, Asia y América Latina, son utilizado como cuerpos receptores de aguas sin tratamiento, ocasionando en el 2012 más de 80.000 muertes por consumo de agua potable contaminada. Aunado a esto, las zonas en mares y océanos muertas por desoxigenación aumentan por el vertido de aguas residuales sin tratar, afectando a $245.000 \mathrm{~km} 2$ de ecosistemas marinos, con repercusiones en las cadenas alimenticias. [1]

El Salvador no escapa a esta situación, en general la calidad de las aguas de los ríos es mala, solo el $17 \%$ del agua superficial es apta para ser potabilizada, ya que casi todas las aguas superan los límites establecidos para una buena calidad ambiental y sanitaria en DBO5 y Coliformes fecales. [2]

Concretamente en la ciudad de La Libertad [3] han expuesto sobre los diversos problemas en el manejo de las aguas residuales, el alcantarillado sanitario y en general de la gestión municipal, que provocan un exceso de contaminación, tal y cómo se corrobora por [4] al encontrar que los límites de DBO DQ05, Aceites y Grasas, Sólidos Suspendidos Totales, sobrepasan los límites máximos permitidos, incrementando la carga orgánica en las aguas residuales especiales, mismas que son el objeto de estudio en esta investigación y cuyo concepto según la norma salvadoreña obligatoria NSO13.49.01:09, se refiere a aguas residuales generadas por actividades agroindustriales, industriales, hospitalarias y todas aquellas que no se consideran de tipo ordinario, es decir, que no sean generadas por actividades domésticas.

La alta carga contaminante que llega a la planta de tratamiento municipal, provoca aumento de los costos de operación y mantenimiento y disminuye su eficiencia en la depuración de las aguas.

\section{Gestión ambiental estratégica y Educación Ambiental}

Según [5] la gestión ambiental estratégica debe incorporarse como punto de partida para lograr identificar todos los aspectos ambientales que tengan la capacidad de generar cierto impacto ambiental adverso o beneficioso y con ello apoyar la consecución de resultados estratégicos propuestos en los planes de acción de las organizaciones.

Dentro de los factores de éxito para lograr una gestión ambiental estratégica se encuentran el compromiso, la organización y el liderazgo, que, para este caso en particular, se asocian al tema de educación ambiental desde una visión que busca transformar para concretar acciones y cambios positivos.

Tal y como lo señalan [6], la educación no formal, no debe limitarse solo a sensibilizar, sino a informar y buscar la participación de los diferentes actores en la resolución de la problemática ambiental. Por tal motivo es necesario promover las conductas pro ambientales, auto reflexionar sobre la conducta y hasta cambio en estilos de vida para impactar positivamente sobre el componente ambiental [7].

Lo anterior se puede abordar desde un modelo ambiental, y tal como lo indican [8], existe una amplia gama de modelos ambientales, contando todos con ventajas y desventajas. Por tal motivo los modelos deben enfocarse en prácticas que permitan abarcar atributos como el conocimiento, las actitudes, barreras, competencias y comportamiento responsable con miras a mejorar la calidad de vida a partir de la solución de problemas cotidianos. 
En este sentido, modelos como el de acción ambiental positiva, el cual enfatiza más en la acción que en el comportamiento, en donde se interrelacionan los conceptos ambientales, las habilidades, actitudes y el empoderamiento para llegar a una acción ambiental positiva, puede resultar relevante para enfrentar problemas cotidianos con la Gestion Integral de las aguas residuales especiales.

De igual forma, la Asociación Norteamericana de Educadores Ambientales, la NAAEE (por sus siglas en inglés) [9], propone que para una alfabetización ambiental es necesario trabajar sobre las competencias, expresadas como las habilidades y destrezas de un individuo o grupo de ellos, y específicamente priorizar en la identificación de problemas ambientales; el análisis de estos problemas, la evaluación de posibles soluciones y la propuesta y justificación de las acciones para hacer frente a problemas ambientales.

Mediante un análisis de conocimiento, actitudes, barreras y competencias, es posible una gestión ambiental estratégica que coadyuve al planteamiento posteriormente de un plan de acción que contribuya a una conducta pro ambiental, desarrollando habilidades, destrezas y conocimientos que promueva un proceso de sensibilización y empoderamiento, lo que derivará en un apropiamiento y aplicabilidad de los conceptos teóricos que se brindan en la educación ambiental [8], lo que sin duda coadyuvará a la gestión integral de las aguas residuales especiales (GIAR).

Según [7], [8] y [10] la protección del ambiente es sinónimo de uso racional de recursos y esto parte de una conducta pro ambiental que le permita a las personas reflexionar sobre su propia conducta y de esta forma realizar acciones positivas respecto al ambiente y en este caso específico sobre la GIAR.

En vista de que la sociedad se enfrenta a diversos problemas que repercuten en ámbitos ambientales, sociales, políticos y económicos es que la GIAR debe enfocarse en la sostenibilidad haciendo partícipes a las comunidades, para que se sensibilicen y empoderen con el fin de mejorar las condiciones socioeconómicas para que la problemática ambiental de las aguas residuales se aborde con conocimiento del medio social, ambiental, económico y cultural para que las acciones sean integrales [7] y [8].

\section{Metodología}

\section{Área de Estudio}

El estudio se realizó en ciudad de La Libertad, El Salvador, en las coordenadas 13²9'17.15" latitud norte y 89¹9'14.19" latitud oeste.

El municipio cuenta con una extensión territorial de $162 \mathrm{~km} 2$, mientras que la ciudad cabecera posee una extensión de 3 km2. El municipio tiene una población total de 40867 habitantes, de los cuales el $32,5 \%$ es urbana y el 52,2\% son mujeres [11] y [12].

\section{Definición de estratos}

Se definieron tres estratos de trabajo a saber: a) Micro y Pequeña Empresa (MYPES), b) Técnico conformado por actores involucrados en la Gestión Ambiental de la ciudad de La Libertad y c) Político constituido por todas las personas del concejo Municipal, y el Alcalde.

Los tres anteriores estratos se definieron por criterio experto, según apreciaciones de la Alcaldía, la Gerencia General, la oficina de gestión ambiental, y expertos externos en desarrollo local. 
Instrumento para recolección de información

Se confeccionaron dos encuestas una para ser aplicada a MYPES y la otra en los dos estratos restantes. Las preguntas permitieron obtener información sobre atributos como conocimientos, actitudes y barreras.

Los instrumentos fueron validados por medio de criterio experto, conformado por cuatro especialistas en educación ambiental y desarrollo local, quienes señalaron diversos cambios y mejoras a los instrumentos.

Una vez validados se aplicó una prueba piloto a 30 personas, mismas que no participaron de la investigación, pero que eran representativas de los estratos, lo anterior para afinar detalles de tiempo y redacción de las preguntas, además de aplicar el alfa de Cronbach para determinar la validez interna de las encuestas.

Las encuestas fueron aplicadas desde junio a noviembre 2017 y por medio de un cálculo de muestra con 95\% de confianza, se determinó que la muestra correspondía a 169 MYPES, no obstante, se logró encuestar a 223 MYPES, lo que eleva el margen de confianza a un 99\%.

Para el caso del estrato Técnico, se aplicó la encuesta a 17 funcionarios y para el Político a14 personas, siendo estos la totalidad de los colaboradores.

\section{Prueba Estadística}

Se utilizaron pruebas no paramétricas de Kruskall -Wallis y Chi Cuadrado con un un p value de 0,05 en el programa Infostat versión 2017 profesional, esto con el fin de determinar diferencias significativas entre los estratos y la dependencia entre atributos y estratos.

\section{Resultados y Discusión}

Seguidamente se muestran los resultados más relevantes de las comparaciones realizadas entre los estratos analizados.

\section{Generalidades de los encuestados}

El cuadro 1 muestra las principales características de la población estudiada. Importante resaltar que en la MYPES 63 de cada 100 entrevistados fueron mujeres, por lo que serían quienes eventualmente asistan a capacitaciones o formen parte de un plan de acción, siendo esto relevante ya que el plan debe contemplar que esta es la población que toma decisiones respecto al vertido de líquidos. Las capacitaciones deben ajustarse a un nivel educativo básico, puesto que la población de MYPES cuenta solamente con estudios de secundaria en el mejor de los casos.

El sector técnico tiene formación universitaria, lo que contribuye a un mejor y mayor debate para proponer acciones concretas respecto a la gestión integral de las aguas residuales.

En la parte Política solamente el $43 \%$ posee estudios formales, y a pesar de que tienen gran voluntad y ganas de sacar el municipio adelante, la carencia de ciertas habilidades y destrezas repercute en que las decisiones que se toman sobre la GIAR no sean las mejores.

Cuadro 1. Características de la población en estudio (datos en porcentaje).

\begin{tabular}{|c|c|c|c|}
\hline Característica & MYPES & Técnico & Político \\
\hline Mujeres & 63 & 30 & 36 \\
\hline Hombres & 37 & 70 & 64 \\
\hline Secundaria completa & 41 & 6 & 14 \\
\hline Universidad completa & 0 & 70 & 43 \\
\hline
\end{tabular}




\section{Conocimientos de la población sobre Gestión Integral de Aguas Residuales}

En términos generales se obtuvo como resultado, que el estrato con mayor conocimiento es el Técnico (61\%), seguido del Político (51\%) y por último MYPES con un 38\%. Los conocimientos sobre la GIAR son de suma importancia ya que, si los individuos cuentan con ellos, podrán reflexionar de manera más puntual sobre su accionar respecto al desarrollo sostenible. Los conocimientos conducen a una conducta ambiental, ya que brindan la capacidad de comprender y evaluar los posibles impactos positivos y negativos que tendrían las acciones realizadas sobre el componente ambiental. El cuadro 2 muestra algunos de los resultados obtenidos

Cuadro 2. Conocimientos más relevantes de GIAR en la muestra de estudio (en porcentaje).

\begin{tabular}{|c|c|c|c|}
\hline & \multicolumn{3}{|c|}{ Estrato de estudio } \\
\hline Conocimiento & MYPES & Técnico & Político \\
\hline Agua Residual & 60,54 & 100,00 & 64,29 \\
\hline Producción Más Limpia & 32,29 & 52,94 & 57,14 \\
\hline Trampas de grasa & 7,17 & 36,36 & 33,33 \\
\hline Planta de Tratamiento & 33,18 & 81,25 & 54,55 \\
\hline
\end{tabular}

Los tres estratos analizados cuentan con conocimientos generales sobre la GIAR, lo cual es valioso ya que los mismos influyen sobre las competencias y por tanto, en como los habitantes de La Libertad puedan realizar acciones en pro del ambiente. Sin embargo, al realizar preguntas de verificación para determinar si el conocimiento que dicen tener lo aplican, es donde se evidencia la necesidad de brindar un acompañamiento para re definir algunos conocimientos, que repercutan en las actitudes, comportamientos, habilidades, destrezas y acciones pro ambientales. Ejemplo de esto es que los encuestados, no tienen certeza de que acciones concretas podrían realizar de Producción Más Limpia $(P+L)$ para favorecer la GIAR, siendo que como máximo solo 12 de cada 100 personas conocen y realizan acciones puntuales de $P+L$.

Para ejemplificar otro aspecto, solo el $50 \%$ de la muestra, conoce las labores de mantenimiento que deben recibir las trampas de grasa, tales como limpieza de grasa y aceite acumulada en la superficie, evacuación de sólidos sedimentados o limpieza de paredes internas de la trampa, razón por la cual, las mismas no funcionan de manera adecuada, ya que la población desconoce los beneficios que pueden brindar para el GIAR.

Al aplicar las pruebas estadísticas, se demuestra que no existen diferencias estadísticamente significativas entre los tres estratos $(p=0,07)$, es decir, en términos globales los conocimientos son similares entre los tres sectores analizados, por lo que ante eventuales capacitaciones se podría abarcar contenidos comunes con los tres grupos. Por otra parte, existencia una relación entre el atributo conocimiento y el estrato $(p<0,05)$, siendo los Técnicos los de mayores conocimientos, esto asociado a su nivel de escolaridad que alcanza el $71 \%$ de formación universitaria completa, respecto a un $40 \%$ de Políticos con este grado académico, lo que indica que, a mayor formación académica, se tiene más capacidad de comprender la repercusión de las acciones que se realicen, para poder redefinir o cambiar las actitudes, sensibilizarse, empoderarse y llegar a tener comportamientos por ambientales.

\section{Actitudes}

Todos los estratos presentan actitudes positivas respecto a la GIAR, por ejemplo, el 92\% considera que la gestión actual de las aguas residuales, está ocasionando un problema ambiental que debido a que se desechan aguas cargadas de grasa o restos de comida 
al alcantarillado o directamente a las calles de la ciudad, a la contaminación de las playas producto de las aguas residuales sin tratamiento, a los malos olores que se dan en la ciudad, entre otros. Respecto a preguntas relacionadas con capacitación un 89\% estaría dispuesto en asistir, ya que consideran que esto impactaría positivamente en la gestión de las aguas residuales. Otro aspecto relevante de destacar, es que el 97\% estaría dispuesto a trabajar en firma conjunta con la municipalidad ya que piensan que el trabajo colaborativo impactaría positivamente en la ciudad.

A pesar de existir actitudes positivas, el problema se presenta al pasar a la acción, ya que, aunque existen conocimientos básicos y actitudes positivas, así como ciertas habilidades y destrezas, los restos de alimentos, grasas y aceites se continúan desechando junto con el agua, las trampas de grasa no reciben mantenimiento y de alguna manera se considera que esto es problema del municipio y no de todas las partes involucradas. Por tal motivo, se está ante comportamientos pro ambientales, mismos que deben ser desarrollados, para que se generen competencias y se conviertan en acciones concretas en beneficio del componente ambiental, puesto que las actitudes son elementos relevantes para definir los comportamientos.Las actitudes se han definido como "sentimientos favorables o desfavorables que se tienen hacia alguna característica del ambiente físico o hacia un problema relacionado con él” [13, p.286]. Es decir, es una valoración positiva o negativa hecha sobre la base de experiencias previas. Según [9], afirman que los diferentes tipos de experiencias parecen fortalecer el desarrollo de sus disposiciones y se ha encontrado que predisponen al individuo para actuar e involucrarse en el proceso de toma de decisiones y resolución de problemas.

La figura 1, muestra la existencia de diferencias significativas $(p=0,03)$ entre los estratos analizados, además de existir una dependencia entre el estrato y la actitud, lo que significa que el estrato Técnico es el que más actitud positiva tiene respecto al componente ambiental, quizás por contar con mayores conocimientos, lo que les brinda herramientas a la hora de tomar decisiones para que sus comportamientos se inclinen más por la protección del componente ambiental.

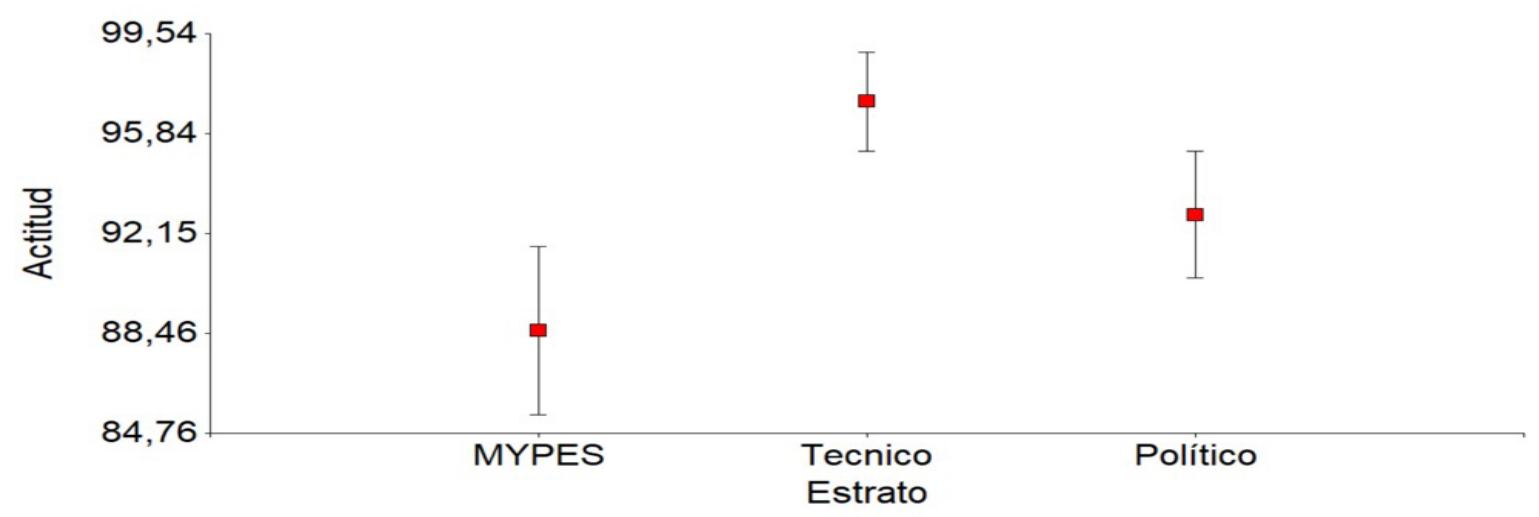

Figura 1. Actitudes respecto a la GIAR 
Los encuestados manifiestan anuencia a participar en charlas sobre manejo de aguas residuales, a separar los residuos sólidos de las aguas, así como colaborar con la municipalidad para que se realicen limpiezas y muestreos para mejorar las medidas de control respecto a la gestión de las aguas residuales, todo esto en proporción de $85 \%$ en todos los estratos. Lo anterior es importante ya que se parte de una base pro ambiental, sin embargo, no se debe perder de vista que, aunque un individuo manifieste que realizará una acción, no se debe dar por sentado que lo hará, ya que la actitud no es equivalente al comportamiento.

\section{Barreras para la GIAR}

Una barrera es un obstáculo, real o no, que una persona puede tener. Se pueden clasificar en tres tipos: las barreras conceptuales, por la noción errónea de los objetivos y alcances de la educación ambiental; las actitudinales, formadas por la percepción de que los educadores tienen actividades más importantes que la educación ambiental; y las barreras de control, que surgen por la percepción de falta de oportunidades, conocimientos, recursos y habilidades. Si estas barreras se presentan, entonces actuarán sobre la sensibilización, los conceptos ambientales, las habilidades, procedimientos y el empoderamiento y el resultado es la falta de incorporación de una cultura ambiental [10].

Los encuestados presentan diversas barreras que deben ser abordadas con el fin de poderlas superar (cuadro 3), pudiendo ser un plan de acción la alternativa para alcanzar esta meta. Los tres estratos manifiestan no conocer con certeza las consecuencias negativas de una inadecuada gestión de las aguas residuales, por lo que en exteriorizan que es necesario adquirir conocimientos para superar esta barrera conceptual.

También manifiestan la existencia de barreras de control, ya que, según los encuestados, una de las fuentes de contaminación excesiva de las aguas es por la mezcla que se da de ésta con restos de comidas, aceites y en general residuos sólidos, por carecer de recipientes y espacios adecuados para realizar una segregación adecuada de cada residuo.

Otra barrera de control que se presenta es la poca coordinación que existe entre los tres estratos analizados, ya que cada quien trabaja por su cuenta sin que exista un acercamiento real entre las partes.

De igual forma se presentan barreras actitudinales como la falta de interés por separar los residuos sólidos de las aguas, lo que provoca la colmatación de las trampas de grasa y del alcantarillado sanitario de la ciudad de La Libertad.

Cuadro 3. Barreras para la GIAR en La Libertad.

\begin{tabular}{|c|c|c|c|c|}
\hline & & \multicolumn{3}{|c|}{ Frecuencia Relativa } \\
\hline Barrera & Tipo & MYPES & Técnico & Político \\
\hline Conocimiento & Conceptual & 59 & 82 & 57 \\
\hline Motivación & Actitudinal & 33 & 71 & 43 \\
\hline Espacio y recipientes & Control & 50 & 82 & 71 \\
\hline Coordinación entre actores involucrados & Control & 50 & 88 & 57 \\
\hline
\end{tabular}

Referente a la anuencia para asistir a capacitaciones la principal barrera de control que se presenta es la falta de tiempo; el sector Político en un 34\% presenta esta barrera, el 63\% de las MYPES lo señala y un 65\% del sector Técnico, por lo que ante la necesidad de realizar capacitaciones dentro de un plan de acción, es necesario tomar en cuenta esta situación para poder superarla. 
La figura 2 muestra la existencia de diferencias significativas respecto a las barreras en los tres estrados $(p=0,02)$, siendo el sector técnico el que presenta en mayor proporción las barreras, esto debido a la criticidad que tienen respecto al tema, y aunque son los que cuentan con mayor conocimiento, apuntan la necesidad de contar con mayores conocimientos para resolver el problema de GIAR en su comunidad. De igual forma apuntan barreras de control, la falta de espacio, tiempo, dinero, o la poca coordinación entre los actores involucrados, por lo que se debe prestar atención a este aspecto, ya que como menciona [14], las barreras de control son más preponderantes que las otras, dado que el desarrollo de conocimientos y habilidades, está supeditado por la dotación de tiempo y recurso monetario a los encargados de incorporar la dimensión ambiental en los programas educacionales, además de esto, las barreras de control actúan sobre el empoderamiento y pertinencia, que de no estar presente se carecerá de la incorporación de la dimensión ambiental.

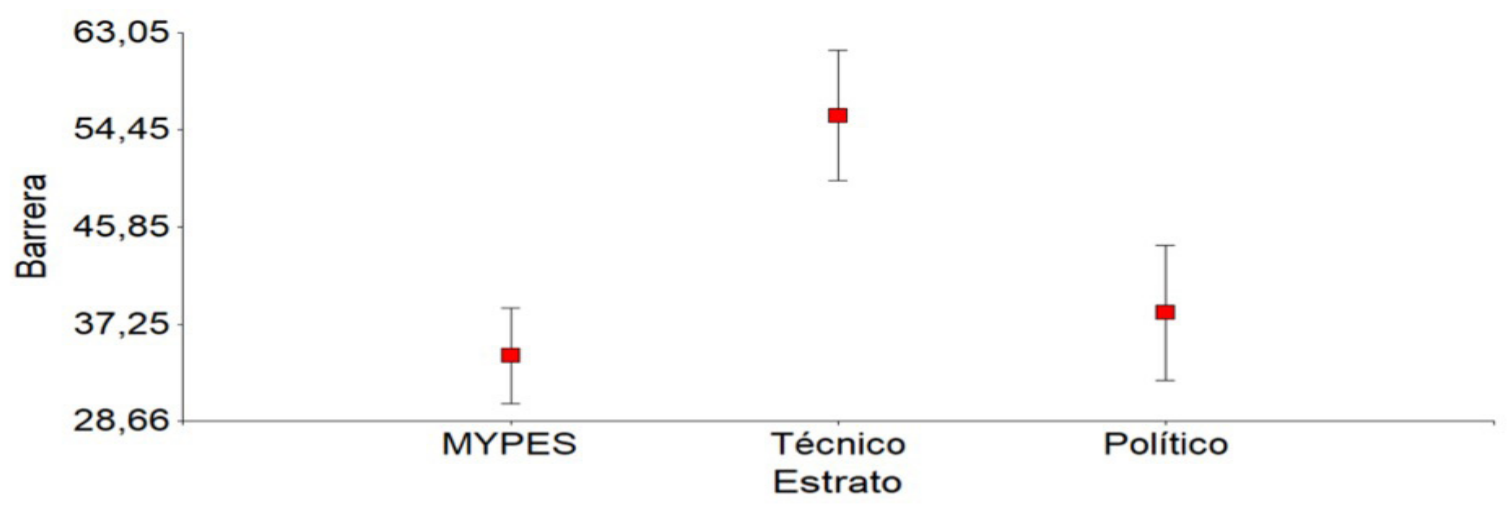

Figura 2. Barreras conceptuales, actitudinales y de control en La Libertad.

\section{Conclusiones}

Para alcanzar una Gestión Integral de las aguas residuales especiales, además de aspectos técnicos y logísticos es necesario incluir a la población y a todos los sectores involucrados como entes preponderantes dentro del proceso. La población en ocasiones realiza acciones que considera adecuadas, porque no cuenta con las habilidades, destrezas, actitudes y conocimientos para entender que sus gestiones perjudican el ambiente y la salud de la población. Por tal motivo, por medio de la educación ambiental, se puede estimular la adquisición o re definición de conocimientos, para que se dé un cambio en los estilos de vida y esto repercuta en la sensibilización y empoderamiento de las personas con el fin de que realicen acciones en pro del ambiente.

En el caso de la ciudad de La Libertad, aunque existen conocimientos por parte de los tres estratos analizados, es necesario profundizar en los mismos y hasta re definirlos, por medio de un proceso de acompañamiento de educación ambiental, ya que en el tanto las personas comprendan e interioricen sus acciones, podrán visualizar el impacto negativo que están ejerciendo sobre el componente ambiental. De acá la necesidad de plantear un plan de acción ambiental en todos los sectores, para el manejo de residuos sólidos y aguas residuales, con énfasis en el sector económico productivo de la ciudad de La Libertad.

Los estratos analizados manifiestan actitudes pro ambientales, pero a pesar de esto continúan presentes los problemas sobre la gestión integral de las aguas residuales y esto se debe a que un individuo puede manifestar su intención de realizar una acción, pero no la ejecuta, esto porque la actitud no es un sinónimo del comportamiento. Por tal motivo, se hace necesario 
un trabajo continuo y colaborativo entre los tres sectores analizados para crear las actitudes por medio de procesos de formación, esto a través de la educación ambiental, que eleven el conocimiento, la afectividad, es decir, conocer las razones por las cuales una persona tiene una conducta determinada y por el último que impacten en la conducta, que se refiere a la intencionalidad de realizar la acción.

Es necesario trabajar para superar las barreras que se identificaron en la investigación, tanto conceptuales, actitudinales y de control. Una vez superadas las barreras las personas podrían impactar con acciones ambientales positivas en pro de una gestión adecuadas de las aguas residuales especiales en La Libertad.

Una vez superadas las barreras las personas podrían impactar con acciones ambientales positivas en pro de una gestión adecuadas de las aguas residuales especiales en La Libertad y así poder mejorar integralmente el sistema de alcantarillado sanitario, mejorar el sistema de trampas de grasa y redes hidráulicas, en las instalaciones internas de todos los negocios generadores de aguas residuales especiales, la instalación de basureros en las principales calles de la ciudad, la creación de ordenanzas municipales en temas de ambiente, la creación de la política ambiental respecto a las aguas residuales especiales, la construcción de una Planta de Tratamiento de Aguas Residuales para la ciudad de La Libertad, y con todo esto poder impactar positivamente contribuyendo en la mejora de la cultura ambiental de la población que coadyuve al de desarrollo turístico de la ciudad y su potencial impacto positivo en el componente ambiental.

\section{Referencias}

[1] WWAP (Programa Mundial de Evaluación de los Recursos Hídricos de las Naciones Unidas). 2017. Informe Mundial de las Naciones Unidas sobre el Desarrollo de los Recursos Hídricos 2017. Aguas residuales: El recurso desaprovechado. París, UNESCO.

[2] MARN (Ministerio de Ambiente y Recursos Naturales). 2016. Recomendaciones para la selección de tratamientos de depuración de aguas residuales urbanas en la República de El Salvador. San Salvador.

[3] F. Rosales, R. Campos, "Gestión y manejo de las aguas residuales no domésticas en la ciudad turística de La Libertad, El Salvador. Desafíos para alcanzar un desarrollo sostenible”, Tecnología en marcha, vol. 32, n 2, pp. 43-53, 2019.

[4] F. Rosales, R. Campos, MD. Rovira, "Aguas residuales no domésticas y su impacto en el desarrollo sostenible: ciudad turística La Libertad, El Salvador”, Tecnología en marcha, vol. 32, nº 3, pp. 135-145, 2019.

[5] INTECO. Sistemas de Gestión Ambiental. Requisitos con orientación para su uso, INTE/ISO 14001:2015.

[6] C. Moreira, F. Araya y C. Charpentier, "Educación ambiental para la conservación del recurso hídrico a partir del análisis estadístico de sus variables" Tecnología en Marcha, vol. 28, nº 3, pp. 75-85, 2015.

[7] R. Campos y M. Camacho, "Factores determinantes para una acción ambiental positiva de la Gestión Integral

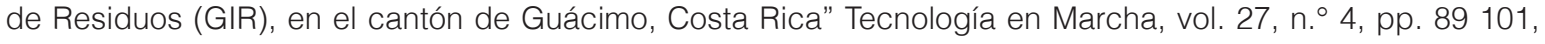
2014.

[8] R. Campos y M. Camacho, "Gestión interuniversitaria y responsabilidad en la gestión ambiental: Plan de acción para el mejoramiento de la gestión integral de los residuos sólidos” Gestión de la Educación, vol. 5, n 2, pp. 1-22, 2015.

[9] K. Hollweg, J. Taylor, R. Bybee, T. Marcinkowski, W. McBeth y P. Zoido, "Developing a framework for assessing environmental literacy. Washington, DC: North American Association for Environmental Education, 2011.

[10] Mata, A., Zúñiga, C., Brenes, O., Carrillo, M., Charpentier, C., Hernández, L. \& Zúñiga, M. (2009). Dimensión Ambiental: Estrategias Innovadoras para la Formación Docente. San José, Costa Rica. CECC.

[11] DIGESTYC, EL Salvador: "Estimaciones y Proyecciones de Población Municipal 2005-2025”, 2014. [En Línea] Disponible en http://www.digestyc.gob.sv/index.php/ novedades/avisos/540-el-salvador-estimaciones-y-proyecciones-de-poblacion.html 
[12] DIGESTYC, "Censo de Población y Vivienda", 2007. [En Línea] Disponible en: http://www.digestyc.gob.sv/ index.php/temas/des/poblacion-y-estadisticas-demograficas/censo-de-poblacion-y-vivienda/poblacion-censos.html

[13] B. Hernández y MC. Hidalgo. "Actitudes hacia el medio ambiente" Psicología Ambiental, Madrid: Pirámide, 2010.

[14] C. Charpentier, "Las barreras para la Educación Ambiental pueden superarse". Biocenosis: revista de educación ambiental. Universidad Estatal a Distancia (UNED). San José, Costa Rica, Vol.18 (1-2), 103-108, 2004. 